\title{
L-CAMP: Extremely local high-performance wavelet representations in high spatial dimension
}

\author{
Youngmi Hur and Amos Ron
}

\begin{abstract}
We study a new wavelet-based methodology for representing data on regular grids. The main attraction of this new L-CAMP methodology is in the way it scales with the spatial dimension, making it, thus, highly suitable for the representation of high dimensional data. The specific highlights of the L-CAMP methodology are three. First, it is computed and inverted by fast algorithms with linear complexity and very small constants. Second, the representation is accompanied by solid mathematical theory that reveals its performance in terms of the maximal level of smoothness that is accurately encoded by the representation. Third, the localness of the representation, measured as the sum of the volumes of the supports of the underlying mother wavelets, is extreme.
\end{abstract}

\section{INTRODUCTION}

The wavelet representation is one of the major representations for data defined on regular grids. There are two main reasons for the popularity of this representation. Firstly, its discrete version is computed and inverted by a fast algorithm, the so-called Fast Wavelet Transform (FWT) [6]. Secondly, it is known to provide optimally sparse representations for the "right type" of functions/datasets (see, e.g., [3], [7]). We refer in this paper to this latter issue as "performance", and actually distinguish between two different types of performance, Jackson-type and Bernstein-type.

We are interested in this paper in wavelet representations in highdimensions. The construction of effective wavelet representations in high spatial dimension is a challenging problem. At a first glance, the choice falls on the so-called tensor-product constructions. These constructions are readily available, their performance is wellunderstood, they are simple, convenient, and, to a degree, computationally effective. However, as the spatial dimension grows, such constructions become immensely non-local in space. Let us illustrate this by the following simple example. Suppose that our construction is in $\mathbb{R}^{5}$, and that we require Jackson-type performance $s=2$, which essentially means that all the wavelets have two vanishing moments. A standard choice would be to use the tensor-product of Daubechies' 4-tap filters [1]. The 5-D tensor-product construction yields 31 mother wavelets. For the case here, the volume of the system, viz., the sum of the support volumes of these 31 mother wavelets is approximately 7500 . This means that every point in space is visited approximately 7500 times by the wavelets within a single scale. That does not sound "local" at all.

In [4], we introduce an algorithm that, for a given fixed performance level $s$ (we deal concretely with $s=2,3,4$ ) and a spatial dimension $n$, yields a wavelet system, to which we refer as an LCAMP system, generated by the mother wavelets $\Psi:=\Psi(n, s) \subset$ $L_{2}\left(\mathbb{R}^{n}\right)$ such that:

Proceedings for SPARS'05 (Rennes, France) talk that will be given by Y. Hur. For a full paper by the authors, see [4].

This work was supported by the US National Science Foundation under Grant ANI-0085984, by the National Institute of General Medical Sciences under Grant NIH-1-R01-GM072000-01, and by the Vilas Foundation at the University of Wisconsin.

Y. Hur is with the Mathematics Department, University of Wisconsin, Madison, WI 53706-1388 USA (e-mail:hur@math.wisc.edu)

A. Ron is with the Computer Sciences Department, University of Wisconsin, Madison, WI 53706-1685 USA (e-mail:amos@cs.wisc.edu)
(1) The performance of the representation matches the given grade $s$. One can choose here to accept Jackson-type performance or to insist on Bernstein-type performance.

(2) The representation can be computed by the FWT, hence with linear complexity.

(3) The representation can be inverted by an algorithm which is different from, and is at least as fast as, the standard inversion of the FWT.

(4) A complete cycle of one decomposition step and its inversion is not only of linear complexity, but the constant in the $O(N)$ bounds, where $N$ is the size of an initial data to be analysed, does not grow with the spatial dimension.

(5) The representation is extremely local.

In the current paper we discuss some of the highlights of the LCAMP methodology, and illustrate the general theory with the aid of two specific constructions. Column 7 of Table I lists the volumes of these two systems. The two volumes satisfy $\operatorname{vol}(\Psi)<5$ (first system), and $\operatorname{vol}(\Psi)<6$ (second system); in both cases, the volume is uniformly bounded independently of the dimension. This should be considered a dramatic improvement over the $\operatorname{vol}(\Psi) \approx 7500$ of the tensor-product construct that was detailed earlier; note also that the volume of all tensor-product constructions (including the Haar system) grows exponentially fast with the dimension.

Given $f: \mathbb{R}^{n} \rightarrow \mathbb{R}$, we denote

$$
f_{j, k}:=D^{j} E^{k} f=2^{j \frac{n}{2}} f\left(2^{j} \cdot-k\right), \quad j \in \mathbb{Z}, k \in \mathbb{Z}^{n} .
$$

Here, $(D f)(t)=2^{n / 2} f(2 t)$ and $\left(E^{k} f\right)(t)=f(t-k)$. We let $\chi$ be the characteristic function of the unit cube, and let $1:=(1, \ldots, 1) \in$ $\mathbb{Z}^{n}$. Furthermore, we let $\mathbf{E}:=\{0,1\}^{n}$, the set of the vertices of the unit cube and let $\mathbf{E}^{\prime}:=\mathbf{E} \backslash 0$.

The outline of the paper is as follows. In Section II, we introduce the L-CAMP systems. In Section III, we present an algorithm for computing and inverting the L-CAMP representation, and examine its complexity. In Section IV, a rigorous discussion of the Jacksontype and Bernstein-type performance of wavelet systems is given together with the main results concerning the performance of LCAMP systems. The proofs can be found in [4].

\section{THE L-CAMP SYSTEM INTRODUCED}

The L-CAMP class in this paper is based on the support function $\phi_{c}:=\chi$ of the unit cube. The function $\phi_{c}$ is refinable with mask $\tau_{c}(\omega)=\prod_{l=1}^{n} \frac{1+e^{-i \omega(l)}}{2}$. Here and later, we use

$$
\omega:=(\omega(1), \ldots, \omega(n)) \in \mathbb{R}^{n}
$$

to denote the generic point in the frequency domain. Let $h_{c}$ be the filter associated with $\bar{\tau}_{c}$ (i.e. $\widehat{h}_{c}=\bar{\tau}_{c}$ ). More general L-CAMP constructions, that use refinable functions $\phi_{c}$ other than $\chi$, are available (see [4] for detailed discussion).

The total number of mother wavelets in our L-CAMP construction is $2^{n}$. Recall that, in $n$-D, the minimal number of mother wavelets is 
$2^{n}-1$, hence that we are slightly redundant. We index the L-CAMP mother wavelets by the set $\mathbf{E}$, i.e., $\psi_{\nu}, \nu \in \mathbf{E}$.

The entire L-CAMP construction is based on two lowpass filters, and on nothing else. One of these filters is used to define the mother wavelet $\psi_{0}$, while the other one is used to define all the remaining mother wavelets. We refer to the first one as the enhancement filter, and to the latter one as the main filter.

We start by selecting the mask $\tau_{e}$ of the enhancement filter. It can be any trigonometric polynomial (in $n$-variables). Initially we require that the mask satisfies the relation

$$
1-\overline{\tau_{e}(2 \omega)} \tau_{c}(\omega)=O\left(|\omega|^{s}\right), \quad \text { near the origin. }
$$

We say that $\tau_{e}$ is of order $s$. While a high $s$ is desired here, we require, at a minimum, that $s \geq 2$. The enhancement filter $h_{e}$ is, then, the filter associated with the mask $\tau_{e}$, i.e., $\tau_{e}=\widehat{h}_{e}$. We further would like the number of taps of $h_{e}$ to be as small as possible. The third, and final, condition that the enhancement filter should satisfy is detailed later.

Next, we define the first mother wavelet, $\psi_{0}$, by the relation

$$
\widehat{\psi}_{0}:=2^{-n / 2}\left(\widehat{\phi}_{c}(\cdot / 2)-\bar{\tau}_{e} \widehat{\phi}_{c}\right) \text {. }
$$

Note that

$$
\psi_{0}=2^{-n / 2}\left(2^{n} \phi_{c}(2 \cdot)-\sum_{k \in \mathbb{Z}^{n}} \overline{h_{e}(k)} \phi_{c}(\cdot+k)\right) .
$$

In order to define the remaining $2^{n}-1$ mother wavelets, we choose a univariate mask $\tau$, denote its univariate filter by $h$, and refer to it as the main filter. The main filter should also satisfy three conditions. The first condition is that it is supported on the odd integers, i.e.,

$$
h(2 m)=0, \quad m \in \mathbb{Z} .
$$

The second condition is that the filter will have high order of polynomial accuracy $N$ : we say that $h$ has accuracy $N$ if

$$
h * P=P, \quad \forall P \in \Pi_{N-1}^{1} .
$$

Recall that the main filter is univariate, hence the accuracy test is conducted on univariate polynomials. A third condition that is required of the main filter is detailed later.

We lift the main filter to $n$-dimensions by aligning it along one of the coordinate axes. There are $n$ different ways to do it, i.e., for $l=1, \ldots, n$,

$$
\tau_{l}(\omega):=\tau(\omega(l))
$$

The mother wavelet $\psi_{\nu}, \nu \in \mathbf{E}^{\prime}$, is defined by the relation

$$
e^{i \nu \cdot \omega} \widehat{\psi}_{\nu}(2 \omega)=2^{-n / 2}\left(1-\overline{\tau_{\lceil\nu\rceil}}(\omega)\right) \widehat{\phi}_{c}(\omega) .
$$

Here \lceil\rceil$: \mathbf{E}^{\prime} \rightarrow\{1, \ldots, n\}$ is a map that determines the orientation of $\tau$ that is assigned to the $\nu$-mother wavelet. While the assignment \lceil\rceil cannot be done at random, there is a great deal of flexibility in choosing it. One way for defining \lceil\rceil goes as follows: for each $\nu \in \mathbf{E}^{\prime}$

$$
\lceil\nu\rceil:=\text { the position of the last 1-digit in the vector } \nu .
$$

For example, for $n=2,\lceil(0,1)\rceil=\lceil(1,1)\rceil=2$ and $\lceil(1,0)\rceil=1$. We extend the domain of the map \lceil\rceil to $\mathbf{E}$ by defining $\lceil 0\rceil:=0$. Under this convention, the valuation map \lceil\rceil of (5) satisfies

$$
\left\lceil\nu-\mathbf{e}_{\lceil\nu\rceil}\right\rceil<\lceil\nu\rceil, \quad \nu \in \mathbf{E}^{\prime} .
$$

Here, $\mathbf{e}_{l}$ is the $l$ th vector in the standard basis for $\mathbb{R}^{n}$.
Note that, if $h$ is $(\lambda-1)$-tap, then $\operatorname{supp} \psi_{\nu}$ is the union of $\lambda$ cubes that are aligned along the $\lceil\nu\rceil$-axis, each of which with volume $2^{-n}$. Defining the volume of $\Psi \subset L_{2}\left(\mathbb{R}^{n}\right)$ to be

$$
\operatorname{vol}(\Psi):=\sum_{\psi \in \Psi} \operatorname{vol}(\operatorname{supp} \psi)
$$

we obtain that the volume of the L-CAMP mother wavelet set

$$
\Psi:=\left\{\psi_{\nu}: \nu \in \mathbf{E}\right\}
$$

is

$$
\operatorname{vol}(\Psi)=\operatorname{vol}\left(\operatorname{supp} \psi_{0}\right)+\frac{\lambda\left(2^{n}-1\right)}{2^{n}} .
$$

That is, the total volume of $\Psi$ is bounded by $\operatorname{vol}\left(\operatorname{supp} \psi_{0}\right)+\lambda$.

We mentioned so far two conditions that we require of the enhancement filter, and of the main filter. Only one additional condition is required here, but it is not as simple as the ones above.

Performance conditions. Our performance analysis of the L-CAMP system is based on the following parameters:

- The order $s \geq 2$ of the enhancement mask (cf. (1)).

- The accuracy $N \geq 2$ of the main filter (cf. (3)).

- The Hölder smoothness $\alpha$ of the $n$-dimensional refinable function $\widetilde{\phi}$ associated with the mask

$$
\tau_{e}\left(\prod_{l=1}^{n} \frac{1+\tau_{l}}{2}\right) .
$$

For Jackson-type performance, we need to assume that

$$
\alpha>0 \text {. }
$$

The performance is then related to $\min \{s, N\}$. In all our concrete constructions, this minimum is $s$.

Bernstein-type performance is related to $\min \{s, N, \alpha\}$, which, again, will coincide in our constructions with $\min \{s, \alpha\}$. So, for this type of performance we desire that

$$
\alpha \geq s,
$$

or at least that $\alpha$ does not lag far behind $s$.

\section{FAST ALGORITHMS FOR COMPUTING AND INVERTING THE REPRESENTATION}

The L-CAMP representation can be computed and inverted by fast algorithms with linear complexity and small constants.

L-CAMP decomposition and reconstruction algorithms. Let $h_{e}$ be the enhancement filter and let $h$ be the main filter. For $l=1, \ldots, n$, let $h_{l}$ be the lifting of $h$ to an $n$-dimensional filter in the $l$-coordinate direction. Let \lceil\rceil be the valuation map from (5). Then:

input $y_{0}: \mathbb{Z}^{n} \rightarrow \mathbb{C}$

(1) Decomposition:

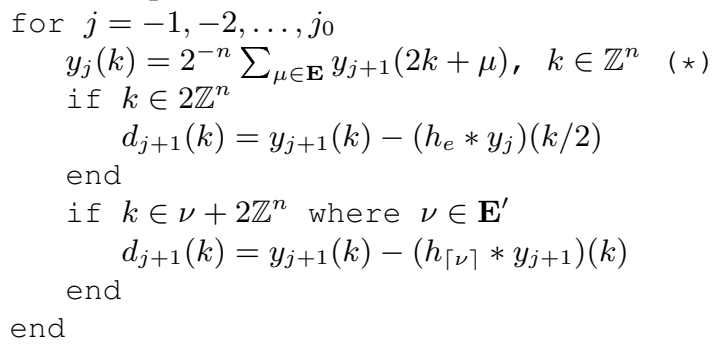

\section{(2) Reconstruction:}

$$
\begin{aligned}
& \text { for } j=j_{0}, \ldots,-1 \\
& \text { if } k \in 2 \mathbb{Z}^{n} \\
& \quad y_{j+1}(k)=d_{j+1}(k)+\left(h_{e} * y_{j}\right)(k / 2)
\end{aligned}
$$




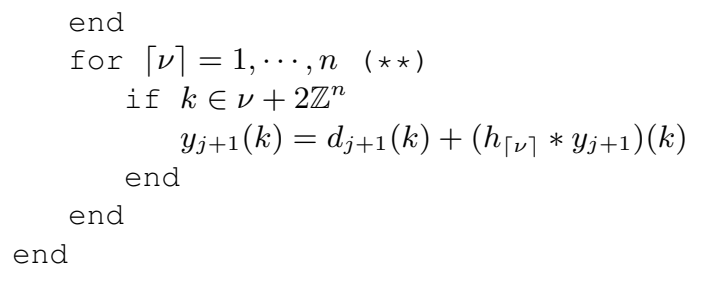

We note that the resulted MRA $\left(y_{j}\right)_{j \leq 0}$ from the line marked by $(*)$ is the MRA associated with $\chi$, that is, assuming $y_{0}(k)=$ $\left\langle f, \chi_{0, k}\right\rangle, k \in \mathbb{Z}^{n}$, for some function $f$, it follows that

$$
y_{j}(k)=2^{j n / 2}\left\langle f, \chi_{j, k}\right\rangle, \quad j<0, k \in \mathbb{Z}^{n} .
$$

After that line, the rest of the decomposition step computes the detail coefficients $d_{j+1}$. The interpretation of the detail coefficients is standard up to normalization, i.e., retaining the same assumption on the initial data $y_{0}$, one proves that for $j<0, \nu \in \mathbf{E}, k \in \mathbb{Z}^{n}$,

$$
d_{j+1}(\nu+2 k)=2^{(j+1) n / 2}\left\langle f,\left(\psi_{\nu}\right)_{j, k}\right\rangle .
$$

The reconstruction step does not resemble its FWT counterpart. The crucial step in the reconstruction is the for loop marked by $(* \star)$. We observe that, if $h_{\lceil\nu\rceil}(l) \neq 0$, then $l$ must be of the form

$$
l=a \mathbf{e}_{\lceil\nu\rceil},
$$

where $\mathbf{e}_{\lceil\nu\rceil}$ is the unit vector in the $\lceil\nu\rceil$-coordinate direction, and $a$ is an odd number. This means that all the values of $y_{j+1}$ that are needed for the computation of $\left(h_{\lceil\nu\rceil} * y_{j+1}\right)(k), k \in \nu+2 \mathbb{Z}^{n}$, lie in $\nu-\mathbf{e}_{\lceil\nu\rceil}+2 \mathbb{Z}^{n}$. Since $\left\lceil\nu-\mathbf{e}_{\lceil\nu\rceil}\right\rceil<\lceil\nu\rceil$ for any $\nu \in \mathbf{E}^{\prime}$ from (6), we have already recovered those values of $y_{j+1}$ previously, hence we are able to compute $y_{j+1}(k)$ as above.

Complexity. We measure complexity by counting the number of "operations" needed in order to fully derive $y_{j}$ and $d_{j+1}$ from $y_{j+1}$, and add the number of operations needed for the inversion. Here, we define "an operation" as the need to fetch an entry from some of our arrays/vectors. Thus, for example, computing one entry in $y_{j}$ from $y_{j+1}$ as in $(*)$ requires $2^{n}$ operations.

Obviously, the complexity here is linear, i.e., $\sim C M$, with $M$ the number of non-zero entries in $y_{0}$, and $C$ some constant. Our goal is to estimate that constant: since $M$ is expected to grow exponentially fast with the dimension, we need, at least, to control very tightly that constant! So, we actually compute the mean number of operations per one single entry in $y_{0}$.

We observe that the number of operations required to process the portion of $y_{j}$ that lies in a cube of lengthsize 2 is about

$$
2^{n}+2\left(1+\text { tap-size of } h_{e}\right)+2 \lambda\left(2^{n}-1\right) .
$$

This means that the cost per entry of performing one complete cycle of decomposition/inversion is bounded by

$$
1+2\left(\lambda+2^{-n}\left(1+\text { tap-size of } h_{e}\right)\right) .
$$

\section{PERformanCE ANALYSIS}

\section{A. The performance of wavelet frames}

Let $\Psi$ be a finite subset of $L_{2}\left(\mathbb{R}^{n}\right)$. The wavelet system generated by the mother wavelets $\Psi$ is the family

$$
X(\Psi):=\left\{\psi_{j, k}: \psi \in \Psi, j \in \mathbb{Z}, k \in \mathbb{Z}^{n}\right\} .
$$

The analysis operator is defined as

$$
T_{X(\Psi)}^{*}: f \mapsto(\langle f, x\rangle)_{x \in X(\Psi)} .
$$

$X(\Psi)$ is a Bessel system if $T_{X(\Psi)}^{*}: L_{2}\left(\mathbb{R}^{n}\right) \rightarrow \ell_{2}(X(\Psi))$ is bounded. A Bessel system $X(\Psi)$ is a frame if the analysis operator is bounded above and below.

Next, we illustrate the way the "performance" of a wavelet frame $X(\Psi)$ may be graded, and use the $L_{2}$-setup to this end. For $\alpha>0$, let $W_{2}^{\alpha}\left(\mathbb{R}^{n}\right)$ be the usual Sobolev space. That is, $W_{2}^{\alpha}\left(\mathbb{R}^{n}\right)$ is the set of functions $f \in L_{2}\left(\mathbb{R}^{n}\right)$ s.t. $|f|_{W_{2}^{\alpha}\left(\mathbb{R}^{n}\right)}:=\left\|\left(|\cdot|^{\alpha} \widehat{f}\right)^{\vee}\right\|_{L_{2}\left(\mathbb{R}^{n}\right)}<\infty$. We would like first the wavelet frame $X(\Psi)$ to satisfy

$$
\sum_{\psi \in \Psi}\left\|T_{X(\psi)}^{*} f\right\|_{\ell_{2}(\alpha)} \leq A_{\alpha}|f|_{W_{2}^{\alpha}\left(\mathbb{R}^{n}\right)}, \quad \forall f \in W_{2}^{\alpha}\left(\mathbb{R}^{n}\right) .
$$

Here,

$$
\left\|T_{X(\psi)}^{*} f\right\|_{\ell_{2}(\alpha)}^{2}:=\sum_{j \in \mathbb{Z}, k \in \mathbb{Z}^{n}} 2^{2 j \alpha}\left|\left\langle f, \psi_{j, k}\right\rangle\right|^{2} .
$$

The supremum of all $\alpha>0$ for which (9) holds is the Jacksontype performance $s_{J}$ of the wavelet frame $X(\Psi)$. It is known that the essential condition $\Psi$ needs to satisfy for having "performancegrade" $s_{J}$ is that each $\psi \in \Psi$ has $s_{J}$ vanishing moments :

$$
\widehat{\psi}=O\left(|\cdot|^{s_{J}}\right), \text { near the origin. }
$$

Another way to measure the performance of $X(\Psi)$ is to insist that, in addition to (9), the inverse inequality holds as well:

$$
\sum_{\psi \in \Psi}\left\|T_{X(\psi)}^{*} f\right\|_{\ell_{2}(\alpha)} \geq B_{\alpha}|f|_{W_{2}^{\alpha}\left(\mathbb{R}^{n}\right)}, \quad \forall f \in L_{2}\left(\mathbb{R}^{n}\right) .
$$

The supremum of all $\alpha>0$ for which (9) and (10) hold is the Bernstein-type performance $s_{B}$ of the wavelet frame $X(\Psi)$. The value of $s_{B}$ is not connected directly to any easy-to-check property of the system $X(\Psi)$. As a matter of fact, the value of $s_{B}$ is related to the smoothness of the dual frame $X\left(\Psi^{d}\right)$, which we now describe.

First, one defines a map $\Psi \ni \psi \mapsto \psi^{d} \in L_{2}\left(\mathbb{R}^{n}\right)$, and extends it naturally to $X(\Psi)$ (i.e., $\left.\left(\psi_{j, k}\right)^{d}:=\left(\psi^{d}\right)_{j, k}\right)$. Assume that $X\left(\Psi^{d}\right)$ is also a frame. The frame $X\left(\Psi^{d}\right)$ is then said to be dual to $X(\Psi)$ if one has the perfect reconstruction property:

$$
f=T_{X\left(\Psi^{d}\right)} T_{X(\Psi)}^{*} f=\sum_{x \in X(\Psi)}\langle f, x\rangle x^{d}, \quad f \in L_{2}\left(\mathbb{R}^{n}\right) .
$$

Here, $T_{X\left(\Psi^{d}\right)}$ is the synthesis operator :

$$
T_{X\left(\Psi^{d}\right)}: \mathbb{C}^{X\left(\Psi^{d}\right)} \ni a \mapsto \sum_{x \in X\left(\Psi^{d}\right)} a(x) x .
$$

Thus, one strives to build wavelet frame systems that have a high number of vanishing moments, and have smooth dual frames. This brings us to the question of how, actually, wavelet systems are constructed. The most general recipe in this regard is known as the Oblique Extension Principle (OEP, [2]). However, in this paper, we will need its special and simpler case, the Unitary Extension Principle (UEP). Both lead to the simultaneous construction of a frame and its dual frame. We describe now the UEP. One uses the tool of MultiResolution Analysis (MRA)([6], [7], [9], [2]): one begins with a refinable function $\phi \in L_{2}\left(\mathbb{R}^{n}\right)$, viz., a function whose Fourier transform satisfies a relation

$$
\widehat{\phi}(2 \cdot)=\tau \widehat{\phi},
$$

for some $2 \pi$-periodic $\tau$ known as the refinement mask. One subsequently defines $V_{0}:=V_{0}(\phi) \subset L_{2}\left(\mathbb{R}^{n}\right)$ to be the closed linear span

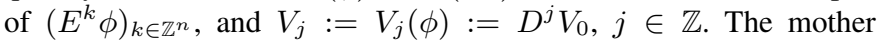
wavelets $\Psi=\left\{\psi_{1}, \ldots, \psi_{r}\right\}$ are then (carefully) selected from $V_{1}$. This implies that every mother wavelet $\psi_{i} \in \Psi$ must satisfy a relation of the form

$$
\widehat{\psi}_{i}(2 \cdot)=\tau_{i} \widehat{\phi},
$$


TABLE I

THE TWO L-CAMP SYSTEMS THAT ARE CONSTRUCTED IN THIS PAPER. COLUMN 1 ENUMERATES THOSE SYSTEMS.

\begin{tabular}{|c|c|c|c|c|c|c|c|c|c|}
\hline L-CAMP & \multicolumn{3}{|c|}{$h_{e}$} & \multirow[t]{2}{*}{$\lambda$} & \multirow{2}{*}{$\begin{array}{c}\text { tap-size of } h_{e} \\
=\operatorname{vol}\left(\operatorname{supp} \psi_{0}\right)\end{array}$} & \multirow[t]{2}{*}{$\operatorname{vol}(\Psi)$} & \multirow{2}{*}{$\begin{array}{l}\text { Hölder smoothness } \\
\text { of } \widetilde{\phi} \geq\end{array}$} & \multirow[t]{2}{*}{$s_{J}$} & \multirow[t]{2}{*}{$s_{B}$} \\
\hline SYSTEMS & -1 & 0 & 1 & & & & & & \\
\hline $\mathrm{I}$ & & $\frac{3}{4}$ & $\frac{1}{4}$ & 3 & 2 & $2+3 \cdot\left(2^{n}-1\right) / 2^{n}$ & 1.4150 & 2 & $\geq 1.4150$ \\
\hline II & $\frac{1}{8}$ & $\frac{1}{2}$ & $\frac{3}{8}$ & 3 & 3 & $3+3 \cdot\left(2^{n}-1\right) / 2^{n}$ & 2.4150 & 2 & 2 \\
\hline
\end{tabular}

for some $2 \pi$-periodic $\tau_{i}$. The dual mother wavelets $\Psi^{d}$ are constructed similarly, using another refinable function $\phi^{d}$ with mask $\tau^{d}$, and corresponding wavelet masks $\left(\tau_{i}^{d}\right)_{i=1}^{r}$.

Suppose now that the two systems $X(\Psi)$ and $X\left(\Psi^{d}\right)$ are known to be, each, a Bessel system, and they satisfy the Mixed Unitary Extension Principle (MUEP) :

$$
\tau(\cdot+\gamma) \overline{\tau^{d}}+\sum_{i=1}^{L} \tau_{i}(\cdot+\gamma) \overline{\tau_{i}^{d}}= \begin{cases}1, & \gamma=0, \\ 0, & \gamma \in\{0, \pi\}^{n} \backslash 0,\end{cases}
$$

and $\widehat{\phi}(0)=\widehat{\phi}^{d}(0)=1$. Then $X(\Psi)$ and $X\left(\Psi^{d}\right)$ form a pair of a wavelet frame and a dual wavelet frame [8]. We refer then to the pair $\left(X(\Psi), X\left(\Psi^{d}\right)\right)$ as a (UEP) bi-framelet.

\section{B. The performance of $L-C A M P$ systems}

We associate here the L-CAMP system with a dual system. We first define the following partial ordering on $\mathbf{E}$ :

$$
\nu^{\prime} \geq \nu \quad \Longleftrightarrow \quad \nu^{\prime}(l)=\nu(l), \quad l=1, \ldots,\lceil\nu\rceil,
$$

where \lceil\rceil is defined as in (5). In particular, $\nu^{\prime} \geq 0$ for all $\nu^{\prime} \in \mathbf{E}$. Given a vector $a \in \mathbb{C}^{n}$, we define

$$
a^{\nu}:=\prod_{l=1}^{n} a(l)^{\nu(l)} .
$$

Lemma 1: For $\nu \in \mathbf{E}$, let $t_{\nu}$ be the wavelet mask that corresponds to the mother wavelet $\psi_{\nu}$ defined in (2) and (4). Let $\xi$ be any trigonometric polynomial such that $\xi(0)=1$ and define a new refinement mask

$$
\tau^{d}:=\tau_{e}(2 \cdot) \tau_{r}\left(1+\xi\left(1-\frac{\tau_{e}(2 \cdot)}{2^{n}} \sum_{\nu \in \mathbf{E}} e_{\nu} \tau^{\nu}\right)\right),
$$

where $\tau_{r}:=\prod_{l=1}^{n}\left(1+\tau_{l}\right) / 2, \tau:=\left(\tau_{1}, \tau_{2}, \ldots, \tau_{n}\right)$, and $e_{\nu}(\omega):=$ $e^{i \nu \cdot \omega}$ for $\omega \in \mathbb{T}^{n}$. We also define dual wavelet masks

$$
t_{\nu}^{d}=2^{-n / 2} e_{-\nu} \sum_{\nu^{\prime} \geq \nu} \boldsymbol{\tau}^{\nu^{\prime}-\nu}\left(1-\xi \tau_{e}(2 \cdot) \tau_{r} e_{\nu^{\prime}}\right), \quad \nu \in \mathbf{E} .
$$

Then the masks $\left(\tau_{c},\left(t_{\nu}\right)_{\nu \in \mathbf{E}}\right)$ and $\left(\tau^{d},\left(t_{\nu}^{d}\right)_{\nu \in \mathbf{E}}\right)$ satisfy the MUEP condition (11), i.e.

$\tau_{c}(\cdot+\gamma) \overline{\tau^{d}}+\sum_{\nu \in \mathbf{E}} t_{\nu}(\cdot+\gamma) \overline{t_{\nu}^{d}}= \begin{cases}1, & \text { if } \gamma=0, \\ 0, & \text { if } \gamma \in\{0, \pi\}^{n} \backslash 0 .\end{cases}$

We are now ready to present our performance analysis of L-CAMP systems. We approach the performance analysis as follows. We first fix an integer $s \geq 2$. We then require the enhancement mask $\tau_{e}$ to be of order $s$ (i.e. to satisfy (1)), and require the main filter $h$ to have accuracy $s$ as well (i.e., to satisfy (3) for $N:=s$ ). Now suppose that we construct the L-CAMP wavelet system using $\tau_{e}$ and $h$ as explained in Section II. Let $\Psi$ be the L-CAMP mother wavelet set as in (7). Then we see that each of the mother wavelets has $s$ vanishing moments: for $\psi_{0}$ this is due to the order of $\tau_{e}$, and for all the other mother wavelets this is due to the accuracy of the main filter $h$.

The other important information needed for the performance analysis of $X(\Psi)$ is the smoothness of the dual system. To this end, we will show that it suffices to know the smoothness of the refinable function $\widetilde{\phi}$, the refinable function associated with the mask $\tau_{e} \tau_{r}$, (cf. (8) and (12)). We note that the standard performance analysis will hinge on the smoothness of the more complicated $\phi^{d}$ associated with $\tau^{d}$ (see, e.g., [7], [5]); thus the reduction of the performance analysis to the smoothness of $\widetilde{\phi}$ is an important step here.

Here we use the usual Hölder smoothness to measure smoothness. For $\eta>0$ non-integer, there is a standard interpretation of what Hölder smoothness $\eta$ means. For positive integers $M=1,2, \cdots$, we say that $f$ has Hölder smoothness $M$ if $f$ has Hölder smoothness $\eta$ for all $0<\eta<M$.

Theorem 1: Let $s \geq 2$ be an integer. Assume that we have an LCAMP system that satisfies (1) for the given $s$, and (3) for $N:=s$. Suppose that $\widetilde{\phi}$ has Hölder smoothness $\eta$ for some $\eta>0$. Then $X(\Psi)$ provides $s_{J} \geq s$ and $s_{B} \geq \min \{s, \eta\}$.

The proof of the above theorem invokes the following lemma, which might be of independent interest.

Lemma 2: Let $s \geq 2$ be an integer. Assume that we have an LCAMP system that satisfies (1) for the given $s$, and (3) for $N:=s$. Suppose that $\widetilde{\phi}$ has Hölder smoothness $\eta$ for some $\eta>0$. Then for every $0<\alpha<\eta$, there exists a wavelet frame $X\left(\Psi^{d}\right)$ associated with a refinable function $\phi^{d}$ that corresponds to the mask $\tau^{d}$ in (12), so that the pair $\left(X(\Psi), X\left(\Psi^{d}\right)\right)$ is a (UEP) bi-framelet and each element in $\Psi^{d}$ has Hölder smoothness $\alpha$.

From Theorem 1 (for any $\eta>0$ and $\eta:=s$ ), we see that the L-CAMP systems satisfying the assumptions in Performance conditions (in Section II) have performance grade at least $s$, in the Jackson sense if we assume (J), and in the Bernstein sense if we assume (B).

In Table I, the two L-CAMP systems that give $s=2$ are presented. For both systems, the main filter $h$ satisfying

$$
h( \pm 1)=\frac{1}{2}, \quad h(m)=0, \text { for } m \neq \pm 1,
$$

is used. L-CAMP systems with higher performance can be found in [4].

\section{REFERENCES}

[1] I. Daubechies, Ten Lectures on Wavelets. Philadelphia, PA: Soc. Ind. Appl. Math., 1992.

[2] I. Daubechies, B. Han, A. Ron, and Z. Shen, "Framelets: MRA-based constructions of wavelet frames," Appl. Comput. Harmon. Anal., vol. 14, no. 1, pp. 1-46, 2003.

[3] R. DeVore, B. Jawerth, and V. Popov, "Compression of wavelet decompositions," Amer. J. Math, vol. 114, no. 4, pp. 737-785, 1992.

[4] Y. Hur and A. Ron, "L-CAMP: Extremely local high-performance wavelet representations in high spatial dimension," 2005, preprint.

[5] G. Kyriazis, "Decomposition systems for function spaces," Studia Math. vol. 157, no. 2, pp. 133-169, 2003.

[6] S. G. Mallat, "A theory for multiresolution signal decomposition: The wavelet representation," IEEE Trans. Pattern Anal. Machine Intell., vol. 11, no. 7, pp. 674-693, 1989.

[7] Y. Meyer, Wavelets and operators. Cambridge: Cambridge University Press., 1992.

[8] A. Ron and Z. Shen, "Affine systems in $L_{2}\left(\mathbb{R}^{d}\right)$ II: dual systems," $J$. Fourier Anal. Appl., vol. 3, pp. 617-637, 1997.

[9] — "Affine systems in $L_{2}\left(\mathbb{R}^{d}\right)$ : the analysis of the analysis operator," J. Funct. Anal., vol. 148, no. 2, pp. 408-447, 1997. 